\title{
The DualBeam FIB in a Materials Science Laboratory
}

\author{
Hendrik O. Colijn
}

Campus Electron Optics Facility, The Ohio State University, 116 W. $19^{\text {th }}$ Ave. Columbus, OH 43210

Focused ion beam instruments have been widely used in the semiconductor industry for sample preparation [1] and are also beginning to show their usefulness in materials science laboratories. The DualBeam FIB/SEM instrument provides many new opportunities for not only sample preparation, but also materials analysis in the materials science environment.

The DualBeam FIB, with the built-in electron column, provides much more flexible preparation possibilities than the single-beam FIB. One useful feature is the ability to directly measure the endpoint of sample thinning by using the penetration of the electron excited volume through the backside of the thin foil. When this occurs, the secondary electron image becomes much brighter and loses contrast (Fig. 1). If we use a $5 \mathrm{kV}$ primary electron beam, the penetration depth is quite small and the foil is sufficiently thin to view in the TEM. This method works regardless of the atomic number and density of the material and is particularly helpful for materials with higher atomic number than $\mathrm{Si}$.

The electron imaging function of the Dual-Beam system also makes possible the "Slice and View" method of visualizing the 3-D structure of a sample. With a single beam instrument, serial sectioning, while possible, would be extremely tedious. By taking electron images of the face of a trench as it is being milled into the sample (Fig. 2.), we can serially reconstruct the 3-D structure (Fig. 3.) [2]. We can correlate the surface and depth features by imaging both the surface and milled faces. Because the ion and electron columns are $52^{\circ}$ apart, tilting the sample surface to $38^{\circ}$ allows us to use the same tilt correction for both the sample and cut surface.

Metals show more defects due to ion damage than do most semiconductor materials. If the ion beam creates a large number of defects, it becomes difficult to separate the defects of interest from those created by the ion beam in both lift-out and H-bar samples (Fig. 4 \& 5). TRIM calculations suggest that the ion damage layer due to both forward and lateral scattering is much less than the TEM sample thickness [3]. At this time it seems that the best remediation is to prepare samples using the $\mathrm{H}$-bar technique followed by a brief low-angle low energy ion polish.

The addition of a STEM detector to the Dual-Beam can reduce the number of samples that go into the TEM. Although the resolution is not as good as a standard materials science TEM, for analytical work it may be sufficient to solve many of the problems which cannot be handled in a regular SEM. Also, by judicious use of milled trenches, one can improve the EDX resolution without having to lift the membrane out of the surface (Fig. 5).

References

[1] L.A. Giannuzzi, F.A. Stevie, Micron 30 (1999) 197.

[2] reconstruction courtesy of Mike Uchic, Wright-Patterson AFB.

[3] B. Prenitzer, Ph. D. dissertation, University of Central Florida, 1999, p. $169 \mathrm{f}$. 


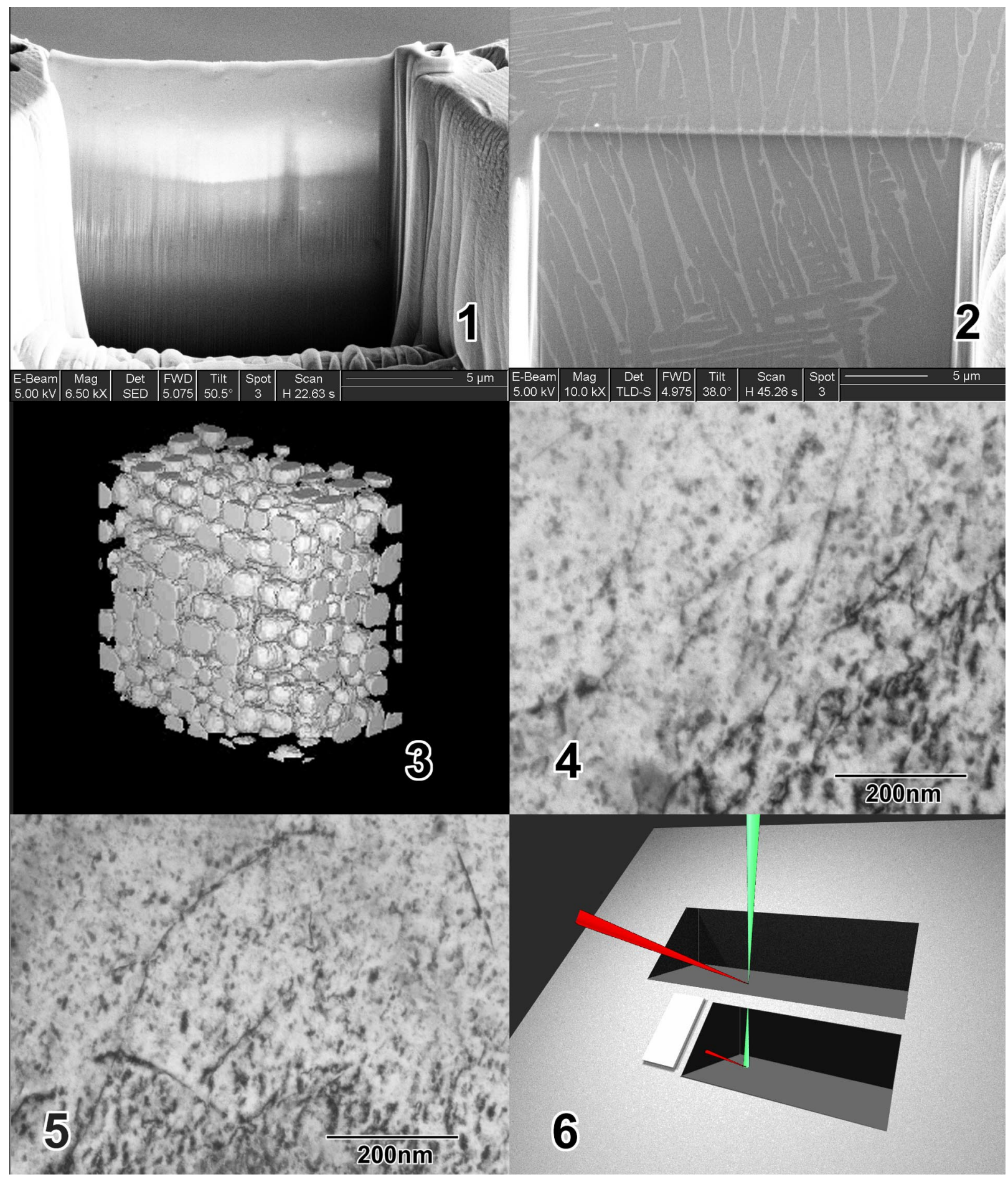

FIG. 1. Electron beam penetration of the thin membrane.

FIG. 2. Section of an alpha/beta Ti alloy .

FIG. 3. Serial reconstruction of gamma prime precipitates in a Ni superalloy.

FIG. 4. Ion damage in alpha Ti using the lift-out technique.

FIG 5. Ion damage in alpha Ti using the H-bar (traditional) technique.

FIG. 6. In-situ membrane technique for improved x-ray resolution. 